\title{
The role of endothelial insulin signaling in the regulation of vascular tone and insulin resistance
}

\author{
David Vicent, ${ }^{1}$ Jacob Ilany, ${ }^{1}$ Tatsuya Kondo, ${ }^{1}$ Keiko Naruse, ${ }^{1}$ Simon J. Fisher, ${ }^{1}$ \\ Yaz Y. Kisanuki, ${ }^{2}$ Sven Bursell, ${ }^{1}$ Masashi Yanagisawa, ${ }^{2}$ George L. King, ${ }^{1}$ \\ and C. Ronald Kahn ${ }^{1}$
}

${ }^{1}$ Research Division, Joslin Diabetes Center and Department of Medicine, Harvard Medical School, Boston, Massachusetts
${ }^{2}$ Howard Hughes Medical Institute, University of Texas Southwestern Medical Center, Dallas, Texas

\begin{abstract}
Insulin receptors (IRs) on vascular endothelial cells have been suggested to participate in insulin-regulated glucose homeostasis. To directly address the role of insulin action in endothelial function, we have generated a vascular endothelial cell IR knockout (VENIRKO) mouse using the Cre-loxP system. Cultured endothelium of VENIRKO mice exhibited complete rearrangement of the $I R$ gene and a more than 95\% decrease in IR mRNA. VENIRKO mice were born at the expected Mendelian ratio, grew normally, were fertile, and exhibited normal patterns of vasculature in the retina and other tissues. Glucose homeostasis under basal condition was comparable in VENIRKO mice. Both eNOS and endothelin-1 mRNA levels, however, were reduced by approximately $30-60 \%$ in endothelial cells, aorta, and heart, while vascular EGF expression was maintained at normal levels. Arterial pressure tended to be lower in VENIRKO mice on both low- and high-salt diets, and on a low-salt diet VENIRKO mice showed insulin resistance. Thus, inactivation of the IR on endothelial cell has no major consequences on vascular development or glucose homeostasis under basal conditions, but alters expression of vasoactive mediators and may play a role in maintaining vascular tone and regulation of insulin sensitivity to dietary salt intake.
\end{abstract}

J. Clin. Invest. 111:1373-1380 (2003). doi:10.1172/JCI200315211.

\section{Introduction}

Insulin receptors (IRs) have been demonstrated on endothelial cells of both large and small blood vessels (1). Insulin binding to its receptor activates both PI3K/AKT and the Ras-MAP kinase pathway. In endothelial cells, the PI3K/AKT pathway mediates an antiapoptotic effect and also results in an increase in gene expression and activation of eNOS (2-4). These effects are enhanced by VEGF and fluid shear stress (5-8).

Multiple hypotheses have been put forward regarding the role of insulin action on vascular tissues as related to glucose homeostasis, control of blood pressure and blood flow, and development of vascular complications. Insulin's effects with regard to vascular complications of diabetes can be considered to be both protective and deleterious. Protective effects of insulin include its ability to protect against apoptosis and to stimulate production of NO $(4,7)$, which results in relaxation of VSMCs and inhibits some of the process-

Received for publication February 6, 2002, and accepted in revised form February 26, 2003.

Address correspondence to: C. Ronald Kahn, Joslin Diabetes Center, One Joslin Place, Boston, Massachusetts 02215, USA. Phone: (617) 732-2635; Fax: (617) 732-2487;

E-mail: c.ronald.kahn@joslin.harvard.edu.

Conflict of interest: The authors have declared that no conflict of interest exists.

Nonstandard abbreviations used: insulin receptor (IR); endothelin-1 (ET-1); vascular endothelial cell insulin receptor knockout (VENIRKO); glucose-tolerance test (GTT); insulintolerance tests (ITT); systolic blood pressure (SBP); mean blood pressure (MBP); diastolic blood pressure (DBP); heart rate (HR); glucose infusion rate (GIR); lipoprotein lipase (LPL). es associated with atherosclerosis. Deleterious effects of insulin are explained by its ability to activate several vascular mediators, such as VEGF, a strong angiogenesis factor, and endothelin-1 (ET-1), a known vasoconstrictor. Hypertension, especially salt-sensitive hypertension, is also associated with insulin resistance (9). In addition, IR action on endothelium has been suggested to play a role in the control of glucose homeostasis in three ways. First, transendothelial transport of insulin is believed to be a rate-limiting step in insulin action on glucose disposal (10). While the exact mechanism(s) of the transendothelial transport in vivo remain debated (11), in endothelial cells in culture insulin transport across the endothelial barrier appears to be mediated by IR (12). Secondly, insulin has shown to act as vasodilator via activation of eNOS in skeletal muscle, and the resultant increase in blood flow has been suggested to contribute significantly to the increase in glucose uptake by skeletal muscle (13). Although the importance of this vasodilatory effect of insulin on whole-body glucose disposal remains controversial, this effect is impaired in individuals with insulin resistance (14-17). Finally, insulin has been suggested to act on endothelium to produce potential mediators of hormone action, such as $\mathrm{NO}$ and $\operatorname{cGMP}(18,19)$. These substances can then diffuse to muscle and fat and act to increase glucose uptake.

To address to what extent insulin action in endothelial cells plays a role in glucose homeostasis and vascular tone, we have generated mice with a specific vascular endothelial cell insulin receptor knockout (VENIRKO). Here, we report the characterization of vascular development, function, and insulin action in 
these animals and explore the role of the insulin receptor in retinal neovascularization.

\section{Methods}

Generation of mice and genotyping. VENIRKO mice were generated using the $C r e-$ loxP system. Mice carrying an $I R$ gene in which exon 4 is flanked by lox sites (20) were bred with a transgenic mice expressing $\mathrm{Cre}$ recombinase under control of the Tie2 promoter-enhancer. Previous studies have shown that Tie2 expression is limited to vascular endothelial cells and the endocardial cushion (21). As a result of the complex breeding, all the mice in these experiments have a mixed genetic background, including contributions from $129 \mathrm{~Sv}, \mathrm{C} 57 \mathrm{Bl} / 6, \mathrm{SJL}, \mathrm{FVB}$, and DBA strains. To minimize the difference of genetic background, we used littermates from same breeding pairs as controls. Genotyping of the mice for the Cre transgene and the floxed IR alleles was performed by genomic PCR, using specific primers that allow identification of the presence or absence of the Tie2-Cre transgene and heterozygosity or homozygosity of the IR gene for the floxed or wild-type allele (22). Animals were housed on a 12-h light/dark cycle and were fed a standard rodent chow. All protocols for animal use and euthanasia were reviewed and approved by the Animal Care Committee of the Joslin Diabetes Center and were in accordance with NIH guidelines. All experiments were carried out in male mice, except as otherwise noted.

Endothelial culture and evaluation of the Cre-mediated recombination of IR in vascular endothelial cells. Two approaches were used to derive microvascular endothelium for primary culture. Cerebrovascular microvessels were isolated by filtration of brain homogenates and digestion with collagenase (23). The digested vessels were filtered sequentially through nylon meshes of three different pore sizes 210,88 , and $55 \mu \mathrm{m}$, respectively. The microvessels retained on 88 - and $55-\mu \mathrm{m}$ filters were plated on $30-\mathrm{mm}$ fibronectin-coated dishes and incubated in DMEM medium containing 10\% FCS and $2 \%$ bovine brain EGF for 7-10 days. Nonendothelial cells were scraped off using a cotton bar under microscopic visualization. Cultures that were considered more than $95 \%$ pure based on morphological examination were used to prepare genomic DNA. Cre-mediated recombination of $I R$ gene was analyzed by PCR using primers surrounding exon 4, as described previously (22).

To obtain enough endothelial cells to prepare RNA for real-time quantitative RT-PCR, primary cultures of endothelial cells were established using cells immunoselected from lungs (24). Briefly, a cell suspension was prepared from lungs by digestion with collagenase (1 $\mathrm{mg} / \mathrm{ml}$ ) for $45 \mathrm{~min}$, followed by filtration using $40-\mu \mathrm{m}$ nylon filters. Endothelial cells were then selected using a rat $\mathrm{Ab}$ to mouse CD-31 (Research Diagnostics Inc., Flanders, New Jersey, USA) coupled to magnetic beads (Dynabeads; Dynal Inc., Lake Success, New York, USA) and coated with sheep anti-rat IgG. Cells were plated on $1 \%$ gelatin-coated $30-\mathrm{mm}$ dishes and grown for 6-7 days in DMEM containing 20\% FCS, 2 mM L-gluta- mine, $2 \mathrm{mM}$ sodium pyruvate, $20 \mathrm{mM}$ HEPES, $1 \%$ nonessential amino acids, $100 \mu \mathrm{g} / \mathrm{ml}$ streptomycin, 100 $\mathrm{U} / \mathrm{ml}$ penicillin, $100 \mu \mathrm{g} / \mathrm{ml}$ heparin, and $100 \mu \mathrm{g} / \mathrm{ml}$ endothelial cell growth supplement (Sigma-Aldrich, St. Louis, Missouri, USA). Cells were then trypsinized and subjected to a second round of selection. For RNA from analysis, approximately $10^{6}$ endothelial cells in each genotype were harvested, and RNA was isolated using an RNeasy mini kit (QIAGEN, Valencia, California, USA) as described by the manufacturer.

Real-time quantitative RT-PCR. Primers and probes for real-time quantitative RT-PCR were designed using Primer Express 1.5a software (ABI Prism; Perkin-Elmer Applied Biosystems, Foster City, California, USA) and were as follows: for $I R, 5^{\prime}$ primer, $5^{\prime}$-CAG AAT GTG ACA GAG TTT GAT GGG-3'; 3' primer, 5'-CGG GTC AAT ATC CAC CAC AGT-3', and probe: 6FAM (carboxyfluorescein)CAG GAT GCT TGT GGC TCC AAC AGC TG-TAMRA ( $N, N, N 9, N 9$-tetramethyl-6-carboxyrhodamine). For $V E G F$, $5^{\prime}$ primer, 5'-GGA GTA CCC CGA CGA GAT AGA GTA-3'; $3^{\prime}$ primer, 5'-AGC CTG CAC AGC GCA TC-3'; and probe, 6FAM-ATC TTC AAG CCG TCC TGT GTG CCG-TAMRA. For $e N O S$, 5' primer, 5'-CGC CAA CGT GGA GAT CAC T-3'; $3^{\prime}$ primer, $5^{\prime}$-ATC AAA GCG GCC ATT TCC T-3'; and probe, 6FAM-AGC TCT GTA TCC AAC ATG GCT GGA CCCTAMRA. For ET-1, 5' primer, $5^{\prime}$-TGG ACA TCA TCT GGG TCA ACA-3'; $3^{\prime}$ primer, $5^{\prime}$-GAC CTG GAA GAA CCT CCC AAT C-3'; and probe, 6FAM-TCC CGA GCG CGT CGT ACC GTATAMRA. Real-time quantitative RT-PCR was done with the TaqMan One-Step RT-PCR Master Mix Reagents kit (Perkin-Elmer Applied Biosystems) as described by the manufacturer. The RT-PCR mixture $(50 \mu \mathrm{l})$ contained 12.5 U of Multiscribe reverse transcriptase, $20 \mathrm{U}$ of RNase inhibitor, $I R$ primers and probe or VEGF primers and probe, eNOS primers and probe, or ET-1 primers and probe, at concentrations of $90-100 \mathrm{nM}$ and $100 \mathrm{nM}$, for each set of primers and probe, respectively, and $5 \mathrm{ng}$ of total RNA template from primary culture of lung endothelial cells. Amplification and detection of specific products was performed with the ABI Prism 7700 sequence detection system (Perkin-Elmer Applied Biosystems) with the following cycle profile: 1 cycle at $48^{\circ} \mathrm{C}$ for $30 \mathrm{~min}, 1$ cycle at $95^{\circ} \mathrm{C}$ for $10 \mathrm{~min}, 40$ cycles at $95^{\circ} \mathrm{C}$ for $15 \mathrm{~s}$, and $60^{\circ} \mathrm{C}$ for $1 \mathrm{~min}$. As an internal control, rodent GAPDH probe and primers (Perkin-Elmer Applied Biosystems) were used for RNA template normalization.

RNA isolation and Northern blot analysis. RNA from heart and aorta of control and VENIRKO mice was isolated using TRI Reagent (Molecular Research Center Inc., Cincinnati, Ohio, USA), and $20 \mu \mathrm{g}$ was subjected to Northern blot analysis using a 650-bp cDNA fragment of rat eNOS (kindly provided by Mark A. Perella and Arthur M.E. Lee, Harvard School of Public Health, Boston, Massachusetts, USA). Quantitation of eNOS mRNA was performed with a PhosphorImager (Molecular Dynamics, Sunnyvale, California, USA) and the levels normalized to those of the 36B4 mRNA.

Metabolic studies. Glucose-tolerance tests (GTTs) were performed in animals that had been fasted overnight for 
$16 \mathrm{~h}$, using an intraperitoneal injection of a $2 \%$ glucose at a dose of $2 \mathrm{~g} / \mathrm{kg}$ body weight. Insulin-tolerance tests (ITTs) were performed in the early afternoon in random-fed animals by intraperatoneal injection of 1.5 $\mathrm{U} / \mathrm{kg}$ body weight of regular human insulin. Blood glucose levels were determined from whole venous blood obtained from the tail using an automatic glucose monitor (One Touch II; Lifescan, Milpitas, California, USA). Insulin concentrations in serum were determined with an ELISA assay using mouse insulin as standard (Crystal Chem Inc. Chicago, Illinois, USA). Serum-free fatty acid and triglyceride concentrations were analyzed immediately after separation of serum by colorimetric enzyme assays, using the NEFA C kit (Wako Chemicals USA Inc., Richmond, California, USA) and the GPOTrinder assay (Sigma-Aldrich), respectively.

Glycemic clamps to assess the kinetics of insulin action. Euglycemic-hyperinsulinemic clamps were performed to evaluate differences in the time course of insulin action. Two intrajugular in-dwelling catheters were placed under anesthesia 4-6 days in advance of the study. The clamps were performed in awake, unanaesthetized mice after a 6-h fast. After basal tail vein blood glucose sampling at time $-20,-10$, and $0 \mathrm{~min}$, insulin was administered intravenously at time 0 as a bolus $(40 \mathrm{mU} / \mathrm{kg})$ and constant infusion $(4 \mathrm{mU} / \mathrm{kg} / \mathrm{min})$. This high dose of insulin was chosen to both suppress endogenous glucose production and stimulate peripheral glucose use. Blood glucose concentrations were measured at 2.5, 5, 7.5, 10, 15, 20, 25, 30, 40,50 , and $60 \mathrm{~min}$. The intravenous infusion of dextrose $(50 \%)$ was adjusted after each blood glucose determination in order to maintain euglycemia (25).

Morphological analysis of retinal microvasculature. Mice (17 days old) were sacrificed with an overdose of sodium pentobarbital and perfused through the heart with 50 mg of FITC-dextran (Sigma-Chemical) dissolved in $1 \mathrm{ml}$ of $10 \%$ buffered formalin (Fisher Scientific Co., Fair Lawn, New Jersey, USA). The eyes were enucleated and fixed in $10 \%$ formalin for $12 \mathrm{~h}$. The retinas were dissected and flat mounted with VECTASHIELD mounting medium (Vector Laboratories, Burlingame, California, USA). Preparations were observed by fluorescence microscopy and photographed using a digital system.

In vivo measurement of blood flow. Mice were anesthetized, and jugular catheters were implanted for fluorescein infusion. The eyes were dilated (1\% tropicamide, Mydriacyl; Alcon Laboratories Inc., Fort Worth, Texas, USA), and a Hamilton syringe containing 10\% sodium fluorescein was connected to the externalized jugular vein catheter. Retinal blood flow was measured using a video fluorescein angiography methodology that has been described in detail previously (26-29).

Blood pressure. Blood pressure was measured in the morning by tail cuff photoelectric plethysmography in restrained, unanesthetized mice using an UR-5000 UEDA system (UEDA Electronic Works Ltd., Tokyo, Japan). Mice were trained to accept the measurement by conducting three mock blood pressure measurements sessions before the actual measurements. Sys- tolic (SBP), mean (MBP), and diastolic blood pressure (DBP), and heart rate in beats per minutes (HR) were obtained as the average of 20-30 measurements that showed stable readings over 10-15 $\mathrm{min}$. In some experiments, mice were fed a diet containing $0.05 \% \mathrm{NaCl}$ for 2 weeks, then shifted to a $8 \% \mathrm{NaCl}$ diet for 2 additional weeks (Harlan Teklad Laboratory, Madison, Wisconsin, USA). BP was measured at days 12 and 13, and an ITT was performed at day 14 of each diet period.

Statistical analysis of data. Data are expressed as means plus or minus SEM. Comparisons of the means were made using the unpaired Student $t$ test. $P$ values smaller than 0.05 were considered statistically significant.

\section{Results}

$V E N I R K O$ mice lack $I R$ and insulin action in endothelium. Endothelial cell-specific inactivation of the IR was achieved by breeding mice carrying an IR gene in which exon 4 was flanked by lox sequences with Tie 2 Cre transgenic mice. Intercrossing the Cre-positive and Cre-negative IR-lox heterozygous mice resulted in offspring of six genotypes: wild-type, IR-lox/+, and the IR-lox/lox, each

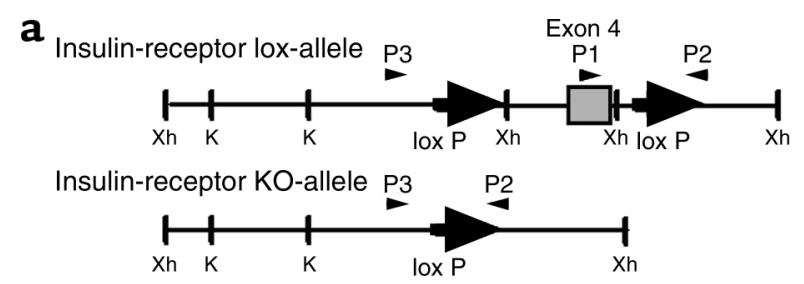

b

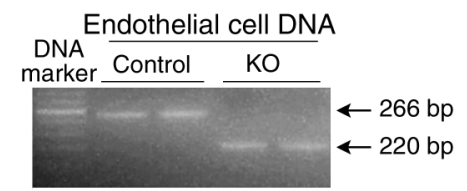

c

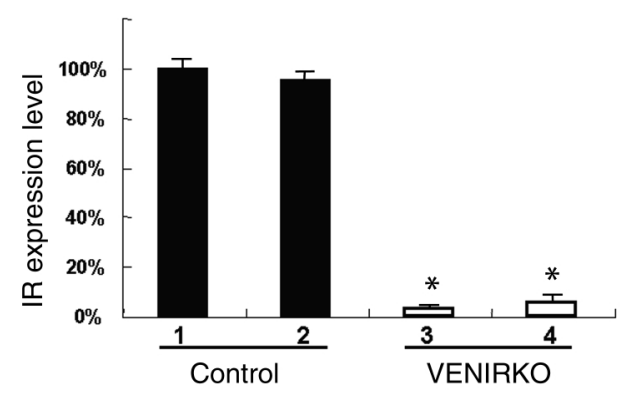

Figure 1

Recombination of the IR gene and expression of the IR mRNA in endothelial cells of VENIRKO mice. (a) Schematic representation of the IR/lox allele showing the position of the primers and the recombined IR knockout allele indicating the deletion of exon 4. (b) The PCR product from control mice corresponds to the $266 \mathrm{bp}$ from the amplification between primers 1 and 2 of the endogenous IR gene allele. The PCR product from VENIRKO mice corresponds to the $220 \mathrm{bp}$ from the amplification between primers 2 and 3. (c) Real-time quantitative RT-PCR analysis to determine the IR expression in RNA extracts from primary cultures of control and VENIRKO mice. The expression level of IR in VENIRKO endothelial cells (lane 3 and 4) were reduced to $3-5 \%$ of control (lane 1 and 2 ). ${ }^{*} P<0.01$ for differences between control and VENIRKO mice. 
a
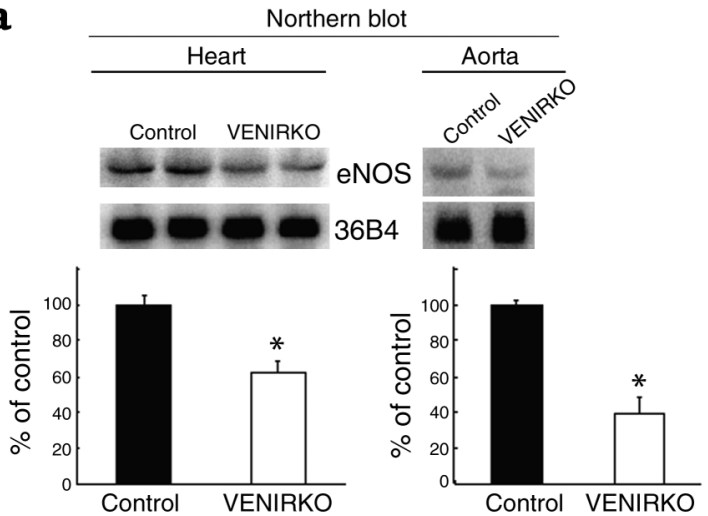

b

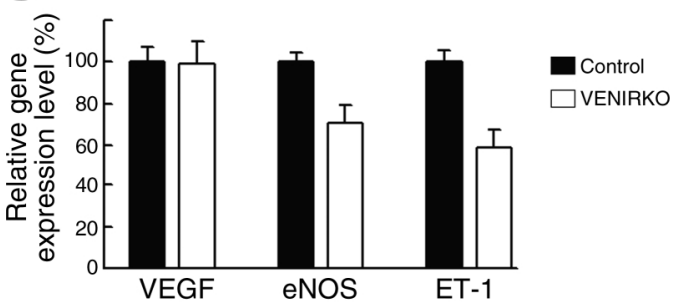

Figure 2

Endothelial mediator gene expression in control and VENIRKO endothelial cells. (a) Northern blot analysis of eNOS in heart and aorta. Total RNA $(20 \mu \mathrm{g})$ from heart and aorta of control and VENIRKO mice ( $n=6$ each) was fractionated and hybridized to cDNA probes for eNOS and 36B4 mRNA. (b) Real-time quantitative RT-PCR analysis to examine the VEGF, eNOS, and ET-1 in RNA extracts from primary cultures. Two independent sets of endothelial cells were prepared from control and two from VENIRKO mice. Each RNA sample was analyzed by real-time quantitative RT-PCR on three independent experiments. ${ }^{*} P<0.05$ for differences between control and VENIRKO mice.

with or without the Cre transgene. During the characterization, no significant phenotypic differences were found among the three genotypes of mice negative for Cre and mice carrying the transgene only, with no $I R-l o x$ allele. Therefore, these four genotypes were combined to a single control group (control), which could be compared with mice positive for the Cre transgene and carrying one (heterozygous) or two lox-IR alleles (VENIRKO).

To assess inactivation of the $I R$ gene, primary cultures of endothelial cells were prepared from brain or lung selected using anti-CD31 $\mathrm{Ab}$, which recognize PECAM-1, a membrane protein specific for endothelial cells, monocytes, leukocytes, and platelets (24). Cremediated recombination of the $I R$ gene in DNA from the endothelial cells was assessed using PCR with three primers, two specific for the intronic sequences upstream and downstream of exon 4 and a third specific for exon 4 (22). A single band of $266 \mathrm{bp}$ was amplified from control, as predicted for the intact $I R$ allele. By contrast, a single band of $220 \mathrm{bp}$ was obtained with cells of VENIRKO mice, indicating complete recombination of the IR-lox allele (Figure 1, a and b).

This was confirmed by real-time quantitative RT-PCR for RNA isolated from primary cultures of endothelial cells, which revealed a 95-97\% reduction in IR mRNA in VENIRKO cells as compared with control (Figure 1c). Using Western blot analysis, there was also little or no detectable IR determined in immunoprecipitates from VENIRKO mice as compared with the wild-type mice (data not shown).

Insulin produces a wide variety of effects in endothelial cells, including stimulation of angiogenesis and vasoregulation. To explore the role of the IR on gene expression in vascular tissues such as heart and aorta, Northern blot analyses were performed using eNOS probes. These revealed a $38.7 \% \pm 7.1 \%$ decrease of eNOS expression in the heart $(P<0.05)$ and $61.8 \% \pm 9.1 \%$ reduction of eNOS mRNA in the aorta $(P<0.05)$ as compared with control (Figure 2a). For better quantitation, we isolated total RNA from cultured endothelial cells and carried out real-time quantitative RT-PCR for VEGF as an angiogentic factor, $e N O S$ as a vasodilator, and ET- 1 as a vasoconstrictor. Two independent sets of endothelial cells were prepared from control and two from VENIRKO mice. Each RNA sample was analyzed by realtime quantitative RT-PCR on three independent experiments. VEGF expression in VENIRKO endothelial cells was comparable to that in control. On the other hand, both eNOS and ET-1 expression in VENIRKO were significantly reduced $30 \% \pm 8 \%(P<0.05)$ and $42 \% \pm 8 \%$ $(P<0.05)$, respectively (Figure $2 \mathrm{~b}$ ).

Structural integrity of vascular tissues and in vivo blood flow. Insulin signaling in endothelial cells activates AKT, and thus insulin could play a role in vascular development, angiogenesis, and endothelial cell apoptosis (4). Inactivation of the IR in endothelial cells, however, did not appear to compromise blood vessel development or
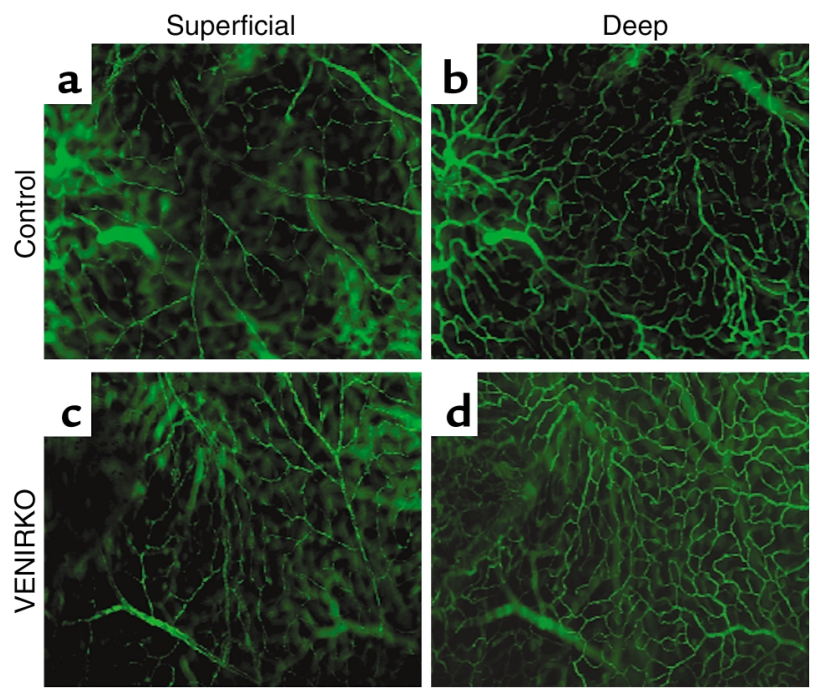

\section{Figure 3}

Flat-mount retinas. Microphotographs $(\times 100)$ of flat-mount retinas prepared from 17-day-old control ( $\mathbf{a}$ and $\mathbf{b})$ and VENIRKO (c and $\mathbf{d}$ ) mice, which had been perfused with fluorescein-conjugated dextran. Microphotographs in a and $\mathbf{c}$ show small arteries and branches of the central artery of the retina. Microphotographs in $\mathbf{b}$ and $\mathbf{d}$ show the deep capillary bed of the same areas shown in a and $\mathbf{c}$. 

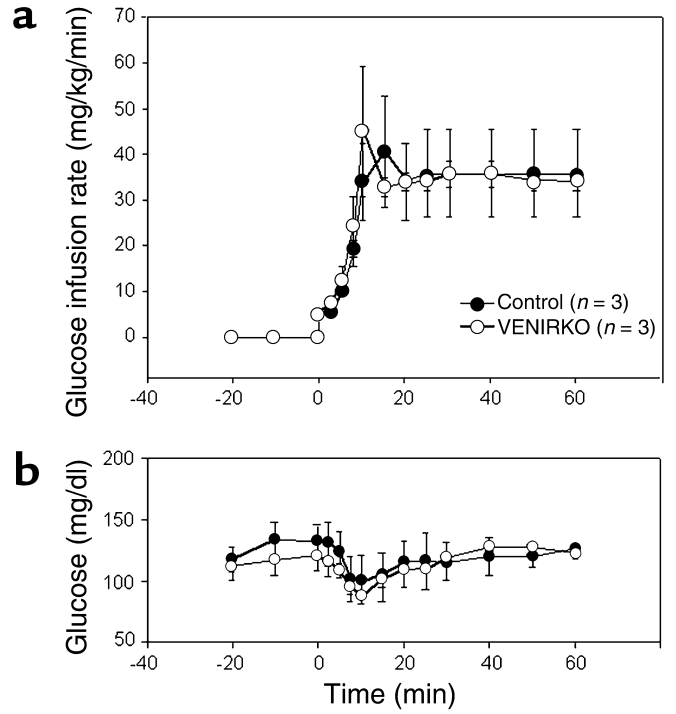

\section{Figure 4}

Evaluation of time course of insulin action in control and VENIRKO mice by euglycemic-hyperinsulinemic clamp. Insulin was administered intravenously at time 0 as a bolus $(40 \mathrm{mU} / \mathrm{kg})$ and constant infusion ( $4 \mathrm{mU} / \mathrm{kg} / \mathrm{min}$ ) for the rest of the experiment. Euglycemia was maintained by glucose infusion. (a) Glucose infusion rates $(\mathrm{mg} / \mathrm{kg} / \mathrm{min}$ ) in control (filled circle, $n=3$ ) and VENIRKO (open circle, $n=3$ ). (b) Blood glucose concentrations during the clamp.

integrity. VENIRKO mice were born at the expected Mendelian frequency, were fertile, showed no defects in growth nor any gross morphological or functional abnormality. Survival up to 24 months of age was not different in VENIRKO mice as compared with control. Furthermore, no change in capillary count or structural alterations were observed in vascular structures of the brain, aorta, and kidney (data not shown). Integrity of capillary bed and small arteries was further examined using flat mount retinas (Figure 3). No abnormal architecture or evidence of compromised capillary integrity could be detected.

Role of IR in kinetics of insulin action in vivo. IRs have been shown to participate in transendothelial transport of insulin in endothelial cells in culture (30); however, whether transendothelial transport of insulin is rate limiting for insulin action in vivo remains controversial (10,
11). To address this issue, we used a modified frequently sampled euglycemic-hyperinsulinemic clamp to assess insulin action. Glucose infusion rates (GIRs) required to maintain euglycemia reflect the kinetic onset and maximal insulin stimulation of glucose turnover in vivo (Figure 4). Fitting GIR to a single two-parameter exponential rise to a maximum curve revealed no significant difference in the half-time for the onset of maximal insulin action ( $6.1 \pm 1.4$ vs $4.4 \pm 1.3 \mathrm{~min}, P=\mathrm{NS})$ nor maximal GIR $(37.7 \pm 2.5$ versus $36.0 \pm 2.6 \mathrm{mg} / \mathrm{kg} / \mathrm{min}$; $P=$ NS). Thus, under this condition, endothelial cell IRs do not appear to play a major rate-limiting role in the access of insulin to metabolically active tissues.

Physiologic effects of IR inactivation in endothelial cells. At 2 months of age, fed and fasting glucose levels, fasting free fatty acid levels, and blood insulin concentrations were comparable in VENIRKO and control mice (Table 1). GTTs and ITTs were also indistinguishable (Figure 5). On the other hand, fasting triglyceride concentrations were reduced in VENIRKO mice approximately $20 \%(P<0.01)($ Table 1$)$ and still slightly lower at 6 months. ITTs at 6 months of age showed that both control and VENIRKO mice had developed mild insulin resistance with a decrease in the response to insulin at 30 and $60 \mathrm{~min}$. Nevertheless, VENIRKO mice remained more glucose tolerant during the GTT (Figure 5).

Effect of knockout of the IR on BP control. Previous studies have demonstrated that insulin has an acute vasodilatory effect (13) and have suggested that insulin resistance at the vascular level may play a role in hypertension. To assess this mechanism, BP and HR were measured at 16 months of age (Figure 6a). The HR in female VENIRKO mice was significantly slower than in control. SBP, MBP, and DBP in male VENIRKO mice were also slightly, but not significantly, lower as compared with control mice on a random diet.

To further explore vascular function and its consequences on insulin sensitivity, mice were placed on lowsalt $(0.05 \% \mathrm{NaCl})$ and high-salt $(8.0 \% \mathrm{NaCl})$ diets for 2 weeks and then reevaluated for BP, HR, and insulin sensitivity. Consistent with the studies on the random diet, SBP, MBP, and DBP were lower in VENIRKO mice than in control on both the low- and high-salt diets. In both groups, BP increased when shifted from low- to

Table 1

Metabolic parameters at 2 and 6 months of age

\begin{tabular}{lcccccc}
\hline & & & & \multicolumn{2}{c}{6 months } \\
& Control & Het & VENIRKO & Control & Het & VENIRKO \\
Weight $(\mathrm{g})$ & $25.9 \pm 0.4$ & $27.0 \pm 0.7$ & $26.3 \pm 0.5$ & $37.3 \pm 0.9$ & $36.3 \pm 1.0$ & $36.9 \pm 1.0$ \\
Fasting glucose $(\mathrm{mg} / \mathrm{dl})$ & $59.3 \pm 1.9$ & $57.2 \pm 3.0$ & $62.7 \pm 3.2$ & $73.4 \pm 2.9$ & $62.3 \pm 1.9 \mathrm{~B}, \mathrm{C}$ & $73.4 \pm 2.8$ \\
Random fed glucose $(\mathrm{mg} / \mathrm{dl})$ & $120 \pm 3$ & $118 \pm 4$ & $123 \pm 4$ & $129 \pm 3.8$ & $126 \pm 4.1$ & $143 \pm 7.9$ \\
Free fatty acids $(\mathrm{mEq} / \mathrm{l})$ & $1.39 \pm 0.03$ & $1.47 \pm 0.05$ & $1.30 \pm 0.07$ & $1.32 \pm 0.05$ & $1.36 \pm 0.05$ & $1.23 \pm 0.06$ \\
Triglycerides $(\mathrm{mg} / \mathrm{dl})$ & $160 \pm 8$ & $180 \pm 10$ & $128 \pm 9 \mathrm{~A}, \mathrm{~B}$ & $133 \pm 7.5$ & $121 \pm 6.6$ & $117 \pm 7.4$ \\
Insulin $(\mathrm{pg} / \mathrm{ml})$ & $317 \pm 35$ & $274 \pm 50$ & $294 \pm 48$ & $619 \pm 104$ & $408 \pm 63$ & $669 \pm 196$ \\
\hline
\end{tabular}

The number of mice in each group for 2-month-old mice were: controls $=41-49$; heterozygous $($ het $)=28-30 ; \mathrm{VENIRKO}=29-30$. The number of mice in each group for 6-month-old mice were: controls $=45-57$, het $=29-30$, VENIRKO $=26-28$. ${ }^{A} P<0.05$ between control and VENIRKO; ${ }^{\mathrm{B}} P<0.05$ between heterozygous and VENIRKO. $c P<0.05$ between controls and heterozygous. 

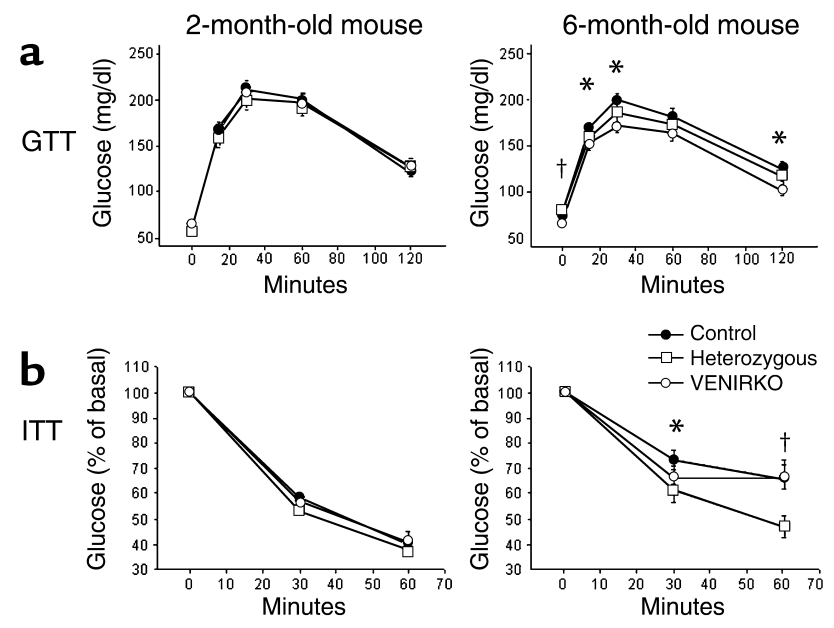

Figure 5

GTTs and ITTs at 2 and 6 months of age. (a) GTT at 2 (left) and 6 (right) months of age in control (filled circle, $n=55$ and 53), heterozygous (open square, $n=30$ and 29), and VENIRKO (open circle, $n=29$ and 27) mice. (b) ITT at 2 (left) and 6 (right) months of age in control ( $n=67$ and 38), heterozygous ( $n=30$ and 25), and VENIRKO ( $n=30$ and 17$)$ mice. ${ }^{*} P<0.05$ and $\dagger P<0.01$ for differences between control and VENIRKO mice.

high-salt diet by approximately $5 \mathrm{mmHg}$, but $\mathrm{BP}$ in the VENIRKO mice remained lower. In addition, HR in VENIRKO mice was significantly slower on the highsalt diet compared with control, consistent with the finding on a regular diet (Figure 6b).

Effects of dietary sodium in insulin sensitivity. BP elevation in response to sodium load is known to cause insulin resistance. To explore the participation of insulin action on endothelial cells in this insulin resistance, we performed ITT on control and VENIRKO mice at the end of the low- and the high-salt diet periods. On a low-salt diet, VENIRKO mice showed significant insulin resistance in comparison with control (Figure 7). When mice were shifted to a high-salt diet, insulin sensitivity in control mice was impaired as expected, whereas in VENIRKO mice, insulin sensitivity improved slightly, but not significantly. This result suggests that insulin signaling in endothelial cells participates in the mechanisms that connect whole-body insulin sensitivity and vascular function.

\section{Discussion}

To address the role of insulin action on vascular tissues in regulation of glucose metabolism and vascular function, we have generated and characterized a VENIRKO mouse. Previous studies have shown that the Tie2 promoter-enhancer construct can direct expression of Cre exclusively in endothelial cell lineage (21). High-efficiency recombination and $I R$ gene inactivation were apparent by genomic PCR and real-time quantitative RT-PCR.

Many of the regulatory effects attributed to endothelial cells, especially in control on vascular tone, depend on their ability to produce NO and ET-1. eNOS, which produces $\mathrm{NO}$, is regulated at the level of expression and activity by insulin $(2,3)$. There is a significant decrease in eNOS mRNA expression in VENIRKO endothelium, indicating a role of insulin in the regulation of eNOS expression in these tissues, even in basal state.

ET-1, on the other hand, is a vasoconstrictive peptide derived from endothelium $(31,32)$. Insulin has been shown to directly stimulate ET-1 gene transcription (33), and VENIRKO endothelial cells show decreased ET-1. Indeed, in endothelial cells, ET-1 expression is reduced even more than eNOS mRNA.

The effect of changes in these vasoactive mediators is complex (34-36). A reduction in eNOS expression would be expected to be associated with an increase in vascular tone and BP. Indeed, a small increase in BP is observed in the eNOS knockout mouse (37). Decreased expression of ET-1, on the other hand, would be expected to increase relaxation of vascular tone and leads to a decrease in BP. Heterozygous inactivation of the ET-1 gene, however, results in hypertension in mice (38), due to an attenuation of parasympathetic activity. Thus, a disruption of the equilibrium between ET-1 and eNOS could lead to either hypertension or hypotension (36). VENIRKO mice have slightly lower BP than control mice and respond normally to high- and low-salt diets, probably as a result of the change in balance of eNOS and ET-1 expression that is critical for maintenance of BP in basal state. To further assess the microvascular function in VENIRKO mice, the response to the iontophoresis of acetylcholine (endothelium dependent) and sodium nitroprusside (endothelium independent) could be done.

Another proposed function of the endothelial IR is facilitation of transport of insulin (12). Our results reveal no alteration in the half-time for insulin stimulation of glucose turnover in VENIRKO mice. Many (10, $39,40)$, but not all $(25,41)$, studies suggest the signal to suppress hepatic glucose production is generated, at least in part, indirectly by insulin action in the periphery, supporting the hypothesis that insulin transport across peripheral endothelium is a critical element determining the kinetics of insulin action in all major insulin-sensitive tissues (39). Moreover, the fact that GIR achieved in this study markedly exceeds basal glucose turnover (41) indicated that insulin crossed capillary endothelium and stimulated glucose uptake and use in peripheral tissues. Thus, in agreement with modeling studies (11), our results do not support a rate-limiting role for the IR in vivo in the transport of insulin across the endothelial barrier in stimulating glucose turnover. Our findings do not, however, exclude the possibility that the IR plays a role in transport of insulin at low insulin concentration or that the IR is a component of insulin transport through the endothelial cells mediating rate-limiting actions into other selected tissues, such as crossing the blood-brain barrier (42).

Despite the absence of IR on endothelial cells, glucose metabolism is comparable in control and VENIRKO mice. While this result would also suggest that insulin action is not critical for endothelial transport in muscle and fat, it is important to recall that glucose toler- 
a

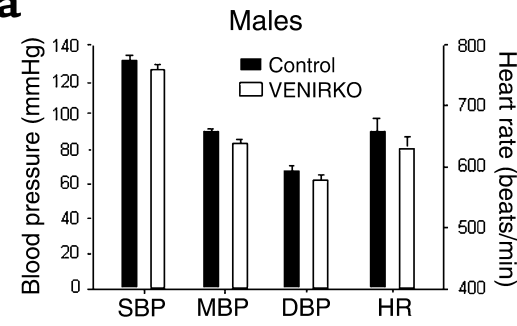

b

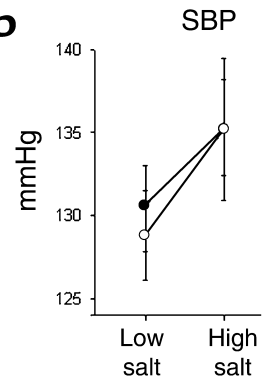

MBP

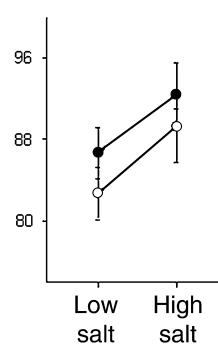

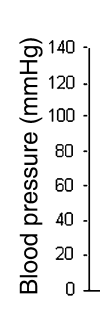

Females

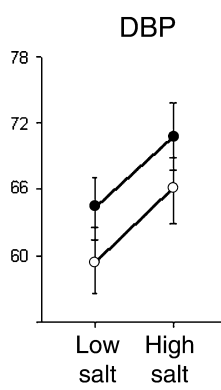

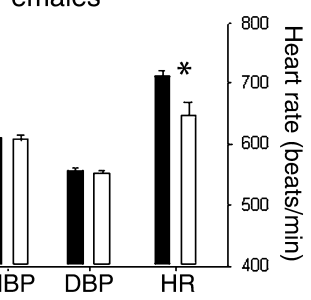

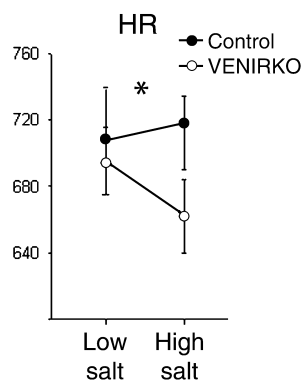

\section{Figure 6}

$\mathrm{BP}$ and HR under basal conditions and low and high $\mathrm{NaCl}$ intake. (a) SBP, MBP, DBP, and $\mathrm{HR}$ in males (left) and females (right) control (black bars) and VENIRKO (white bars) mice. ${ }^{*} P<0.05$ for differences between mean values in control ( $n=20$ males and $n=11$ females) and VENIRKO ( $n=13$ males and $n=5$ females). (b) SBP, MBP, DBP, and HR under low- and high-salt diet in male control (filled circle, $n=12$ ) and VENIRKO (open circle, $n=12$ ) mice. ${ }^{*} P<0.05$ for differences in mean values between control and VENIRKO mice. ance in the muscle- or fat-specific $I R$ knockout mice is normal $(20,43)$. Thus a selective impairment of insulin action at these tissues may not be sufficient to cause diabetes. On the other hand, significant alterations in glucose tolerance are observed in mice with a liver-specific IR knockout (25) or a knockout of Glut4 in muscle (44) or fat (45). The difference in severity of glucose intolerance between the muscle IR and muscle Glut4 knockouts could also be consistent with the suggestion that insulin increases glucose uptake in muscle indirectly by producing vasodilatation (13) or by generating NO and cGMP that can stimulate glucose uptake by the myocytes $(18,19)$. Again, however, the finding of normal glucose tolerance in the VENIRKO mice indicates that these are not major mechanisms in control of blood glucose in vivo.

Serum triglyceride levels in VENIRKO mice are lower than control at 2 months of age and remain stable at 6 months, while control mice show a decrease in triglyceride concentration between 2 and 6 months. Free fatty acid concentrations in VENIRKO mice also tend to be reduced at 2 and 6 months, although this is not statistically significant. Clearance of triglyceride from the blood stream requires the action of lipoprotein lipase (LPL) on chylomicrons and very low-density lipoproteins at the luminal surface of the vascular endothelium. The expression of LPL in myocytes and its translocation to the luminal surface of the endothelial cell are both insulinrelated processes (46). In this context, VENIRKO mice should have shown reduced LPL activity in endothelium and have higher triglyceride levels. Since they did not, it is possible that the expression of LPL in adipocytes or myocytes was enhanced and compensated for the LPL reduction in endothelial cells, that there is another LPL activation pathway in endothelial cells in an insulinindependent manner, or that endogenous production of triglyceride from liver and adipocyte might be reduced. Further investigation is necessary to address these issues.
To further explore the relationship between insulin sensitivity and vascular function, we challenged the mice with low- and high-salt diets. High-salt diets have been shown to produce insulin resistance, especially in those who respond with a marked increase in arterial pressure. As expected, insulin sensitivity in control mice tends to deteriorate from the low- to the high-salt diet. In contrast, VENIRKO mice show insulin resistance on a low-salt diet that remains unchanged when mice are shifted to a high-salt diet, suggesting that insulin signaling in endothelial cells may interact with regulatory mechanisms activated under low-salt diet conditions, such as the renin-angiotensin system, and regulate whole -body insulin sensitivity (47-49).

In conclusion, the endothelial cell $I R$ knockout mouse shows normal glucose homeostasis and normal vascular development, but altered ET-1 and eNOS expression and modest alteration in regulation of BP and insulin sensitivity on a low-salt diet. The existence of counterregulatory mechanisms may compensate for the deficiency of insulin effect, or insulin's role may be dispensable in metabolism and cardiovascular function.
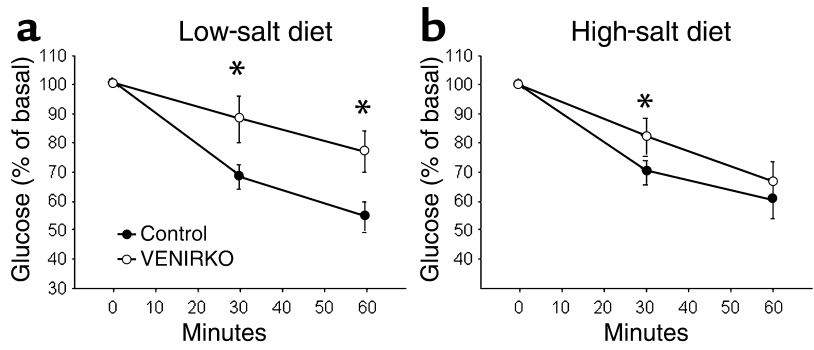

\section{Figure 7}

ITTs under dietetic low and high $\mathrm{NaCl}$ intake. ITT in control (filled circle, $n=7$ ) and VENIRKO (open circle, $n=6$ ) mice. Under low-salt $\operatorname{diet}(\mathbf{a})$ and high-salt diet (b). ${ }^{*} P<0.05$ for differences in mean values between control and VENIRKO mice. 


\section{Acknowledgments}

The authors gratefully thank Islay Campbell, Karrie Miller, and Gwenivere Lovewell for expert technical assistance in the metabolic studies and mice genotyping, and Allen C. Clermont who performed the retinal blood flow and blood pressure measurements. We are indebted to Francis W. Luscinskas and Yaw-Chyn Lim for their advice and guidance in the primary culture of mice endothelial cells, to Kiyoshi and Izumi Suzuma for help with the morphological studies and Efi Kokkotou for expert advice in real-time quantitative RT-PCR. We are grateful to Julie Marr for secretarial assistance in the preparation of the manuscript. This work was supported by a grant from the Manpei Suzuki Diabetes Foundation of Japan, NIH grant DK-31036 and DK-33201, and the Joslin Diabetic Endocrinology Research Center.

1. Jialal, I., et al. 1985. Characterization of the receptors for insulin and the insulin-like growth factors on micro- and macrovascular tissues. Endocrinology. 117:1222-1229.

2. Kuboki, K., et al. 2000. Regulation of endothelial constitutive nitric oxide synthase gene expression in endothelial cells and in vivo: a specific vascular action of insulin. Circulation. 101:676-681.

3. Zeng, G., et al. 2000. Roles for insulin receptor, PI3-kinase, and Akt in insulin-signaling pathways related to production of nitric oxide in human vascular endothelial cells. Circulation. 101:1539-1545.

4. Hermann, C., Assmus, B., Urbich, C., Zeiher, A.M., and Dimmeler, S. 2000 Insulin-mediated stimulation of protein kinase Akt: A potent survival signaling cascade for endothelial cells. Arterioscler. Thromb. Vasc. Biol. 20:402-409

5. Dimmeler, S., Assmus, B., Hermann, C., Haendeler, J., and Zeiher, A.M. 1998. Fluid shear stress stimulates phosphorylation of Akt in human endothelial cells: involvement in suppression of apoptosis. Circ. Res. 83:334-341.

6. Dimmeler, S., et al. 1999. Activation of nitric oxide synthase in endothelial cells by Akt-dependent phosphorylation. Nature. 399:601-605.

7. Gerber, H.P., et al. 1998. Vascular endothelial growth factor regulates endothelial cell survival through the phosphatidylinositol 3'-kinase/Akt signal transduction pathway. Requirement for Flk-1/KDR activation. J. Biol. Chem. 273:30336-30343.

8. Gallis, B., et al. 1999. Identification of flow-dependent endothelial nitricoxide synthase phosphorylation sites by mass spectrometry and regulation of phosphorylation and nitric oxide production by the phosphatidylinositol 3-kinase inhibitor LY294002. J. Biol. Chem. 274:30101-30108.

9. Ogihara, T., et al. 2001. Insulin resistance with enhanced insulin signaling in high-salt diet-fed rats. Diabetes. 50:573-583.

10. Yang, Y.J., Hope, I.D., Ader, M., and Bergman, R.N. 1989. Insulin transport across capillaries is rate limiting for insulin action in dogs. J. Clin. Invest. 84:1620-1628.

11. Steil, G.M., Ader, M., Moore, D.M., Rebrin, K., and Bergman, R.N. 1996. Transendothelial insulin transport is not saturable in vivo. No evidence for a receptor-mediated process. J. Clin. Invest. 97:1497-1503.

12. King, G.L., and Johnson, S.M. 1985. Receptor-mediated transport of insulin across endothelial cells. Science. 227:1583-1586.

13. Baron, A.D. 1994. Hemodynamic actions of insulin. Am. J. Physiol. 267:E187-E202.

14. Baron, A.D. 1993. Cardiovascular actions of insulin in humans. Implications for insulin sensitivity and vascular tone. Baillieres Clin. Endocrinol. Metab. 7:961-987.

15. Natali, A., et al. 1994. Insulin resistance and vasodilation in essential hypertension. Studies with adenosine. J. Clin. Invest. 94:1570-1576.

16. Andersson, P., Lind, L., Berne, C., Berglund, L., and Lithell, H.O. 1999. Insulin-mediated vasodilation and glucose uptake are independently related to fasting serum nonesterified fatty acids in elderly men. J. Intern. Med. 246:529-537.

17. Mather, K., Laakso, M., Edelman, S., Hook, G., and Baron, A. 2000. Evidence for physiological coupling of insulin-mediated glucose metabolism and limb blood flow. Am. J. Physiol. Endocrinol. Metab. 279:E1264-E1270.

18. Etgen, G.J., Jr., Fryburg, D.A., and Gibbs, E.M. 1997. Nitric oxide stimulates skeletal muscle glucose transport through a calcium/contraction- and phosphatidylinositol-3-kinase-independent pathway. Diabetes. 46:1915-1919.

19. Young, M.E., Radda, G.K., and Leighton, B. 1997. Nitric oxide stimulates glucose transport and metabolism in rat skeletal muscle in vitro. Biochem. J. 322:223-228.

20. Bruning, J.C., et al. 1998. A muscle-specific insulin receptor knockout exhibits features of the metabolic syndrome of NIDDM without alter- ing glucose tolerance. Mol. Cell. 2:559-569.

21. Kisanuki, Y.Y., et al. 2001. Tie2-Cre transgenic mice: a new model for endothelial cell-lineage analysis in vivo. Dev. Biol. 230:230-242.

22. Kulkarni, R.N., et al. 1999. Tissue-specific knockout of the insulin receptor in pancreatic beta cells creates an insulin secretory defect similar to that in type 2 diabetes. Cell. 96:329-339.

23. Diglio, C.A., Grammas, P., Giacomelli, F., and Wiener, J. 1982. Primary culture of rat cerebral microvascular endothelial cells. Isolation, growth, and characterization. Lab. Invest. 46:554-563.

24. Dong, Q.G., et al. 1997. A general strategy for isolation of endothelial cells from murine tissues. Characterization of two endothelial cell lines from the murine lung and subcutaneous sponge implants. Arterioscler. Thromb. Vasc. Biol. 17:1599-1604.

25. Michael, M.D., et al. 2000. Loss of insulin signaling in hepatocytes leads to severe insulin resistance and progressive hepatic dysfunction. Mol. Cell. 6:87-97.

26. Takagi, C., et al. 1995. Reversal of abnormal retinal hemodynamics in diabetic rats by acarbose, an alpha-glucosidase inhibitor. Curr. Eye Res. 14:741-749

27. Ishii, H., et al. 1996. Amelioration of vascular dysfunctions in diabetic rats by an oral PKC beta inhibitor. Science. 272:728-731.

28. Takagi, C., et al. 1996. Endothelin-1 action via endothelin receptors is a primary mechanism modulating retinal circulatory response to hyperoxia. Invest. Ophthalmol. Vis. Sci. 37:2099-2109.

29. Bursell, S.E., et al. 1997. Specific retinal diacylglycerol and protein kinase C beta isoform modulation mimics abnormal retinal hemodynamics in diabetic rats. Invest. Ophthalmol. Vis. Sci. 38:2711-2720.

30. Baron, A.D., et al. 1995. Insulin resistance after hypertension induced by the nitric oxide synthesis inhibitor L-NMMA in rats. Am. J. Physiol. 269:E709-E715

31. Yanagisawa, M., et al. 1988. A novel potent vasoconstrictor peptide produced by vascular endothelial cells. Nature. 332:411-415.

32. Yanagisawa, M., Kurihara, H., Kimura, S., Goto, K., and Masaki, T. 1988. A novel peptide vasoconstrictor, endothelin, is produced by vascular endothelium and modulates smooth muscle $\mathrm{Ca} 2+$ channels. J. Hypertens. Suppl. 6:S188-S191.

33. Oliver, F.J., et al. 1991. Stimulation of endothelin-1 gene expression by insulin in endothelial cells. J. Biol. Chem. 266:23251-23256.

34. Loennechen, J.P., Stoylen, A., Beisvag, V., Wisloff, U., and Ellingsen, O. 2001. Regional expression of endothelin-1, ANP, IGF-1, and LV wall stress in the infarcted rat heart. Am. J. Physiol. Heart Circ. Physiol. 280:H2902-H2910.

35. Kobayashi, T., et al. 2000. Corresponding distributions of increased endothelin-B receptor expression and increased endothelin- 1 expression in the aorta of apolipoprotein E-deficient mice with advanced atherosclerosis. Pathol. Int. 50:929-936.

36. Cardillo, C., et al. 1999. Insulin stimulates both endothelin and nitric oxide activity in the human forearm. Circulation. 100:820-825.

37. Shesely, E.G., et al. 1996. Elevated blood pressures in mice lacking endothelial nitric oxide synthase. Proc. Natl. Acad. Sci. U. S. A. 93:13176-13181.

38. Kurihara, Y., et al. 1994. Elevated blood pressure and craniofacial abnormalities in mice deficient in endothelin-1. Nature. 368:703-710.

39. Poulin, R.A., Steil, G.M., Moore, D.M., Ader, M., and Bergman, R.N. 1994. Dynamics of glucose production and uptake are more closely related to insulin in hindlimb lymph than in thoracic duct lymph. Diabetes. 43:180-190.

40. Giacca, A., et al. 1992. Importance of peripheral insulin levels for insulininduced suppression of glucose production in depancreatized dogs. J. Clin. Invest. 90:1769-1777.

41. Fisher, S.J., and Kahn, C.R. 2003. Insulin signaling is required for insulin's direct and indirect action on hepatic glucose production. J. Clin. Invest. 111:463-468. doi:10.1172/JCI200316426.

42. Baura, G.D., et al. 1993. Saturable transport of insulin from plasma into the central nervous system of dogs in vivo. A mechanism for regulated insulin delivery to the brain. J. Clin. Invest. 92:1824-1830.

43. Bluher, M., et al. 2002. Adipose tissue selective insulin receptor knockout protects against obesity and obesity-related glucose intolerance. Dev. Cell. 3:25-38.

44. Zisman, A., et al. 2000. Targeted disruption of the glucose transporter 4 selectively in muscle causes insulin resistance and glucose intolerance. Nat. Med. 6:924-928.

45. Abel, E.D., et al. 2001. Adipose-selective targeting of the GLUT4 gene impairs insulin action in muscle and liver. Nature. 409:729-733.

46. Knutson, V.P. 2000. The release of lipoprotein lipase from 3T3-L1 adipocytes is regulated by microvessel endothelial cells in an insulindependent manner. Endocrinology. 141:693-701.

47. Velloso, L.A., et al. 1996. Cross-talk between the insulin and angiotensin signaling systems. Proc. Natl. Acad. Sci. U. S. A. 93:12490-12495.

48. Rao, R.H. 1994. Effects of angiotensin II on insulin sensitivity and fasting glucose metabolism in rats. Am. J. Hypertens. 7:655-660.

49. Richey, J.M., Ader, M., Moore, D., and Bergman, R.N. 1999. Angiotensin II induces insulin resistance independent of changes in interstitial insulin. Am. J. Physiol. 277:E920-E926. 